\title{
An Afferent Hippocampal Fiber System in the Fornix of the Monkey
}

\author{
CHARLES L. VOTAW AND EDWARD W. LAUER ${ }^{1}$ \\ Department of Anatomy, The University of Michigan, Ann Arbor, Michigan
}

In a previous investigation (Votaw, '60b) it was noted that, in spite of massive bilateral removal of the hippocampal formation (Ammon's horn and dentate gyrus), there was a considerable percentage of the $\mathrm{fi}$ bers in the fornix, anterior to the hippocampal commissure, that did not degenerate. This would imply, since the lesions to all intents and purposes eliminated all possible fibers going into the fornix from the region of the temporal lobe, the presence of fibers in the fornix which are afferent with respect to the hippocampus, and further, that these fibers are not commissural in nature. In another study (Votaw, '60a), it was shown that lesions in the septal area would produce a degeneration which could be followed from the area of the lesion in a caudal direction, coursing through the body of the fornix, from which it would enter the fimbria and alveus. The final termination could not be determined with certainty.

There is accumulating in the current literature a considerable body of evidence concerning a hippocampal afferent system in the fornix (see Discussion). Some of this information is gained from morphological experimentation, but the majority of the more suggestive evidence is based on physiological data. To the authors' knowledge there is no information available with respect to the monkey about this fiber system, except that quoted above (Votaw, '60a). In an attempt to investigate more fully the existence of such a fiber system in monkeys, and, if possible, to describe some of its relations, the present study was undertaken.

\section{MATERIALS AND METHOD}

Fourteen monkeys (Macaca mulatta) were used in these experiments. The animals were unselected with respect to sex, and weighed from 2.2 to $5.3 \mathrm{kgs}$. Seven were used only for this investigation and seven others were used as well in another investigation on the function of the hippocampus and are included in this study because the lesions placed were ideally suited for comparison with the former seven cases.

All animals were subjected to aseptic surgical procedures. The only surgical procedure on seven was the production of the lesions to be reported here; lesions were placed in the remaining seven after other studies had been carried out. General anesthesia was used and consisted of ether, sodium pentobarbital ( $20-25 \mathrm{mg} / \mathrm{kg}$ of body weight given intravenously) or a combination of the two anesthetics, ether being used to supplement a somewhat lower dose of sodium pentobarbital. Following closure of the surgical wounds, the animal was given a dose of penicillinchloramphenicol intramuscularly and returned to an isolated cage. The monkeys were observed daily, and additional antibiotics given if it was thought necessary. Wound infection was limited to one case, and a post-operative diarrhea was evident in two others. These complications were easily controlled.

In four of the animals, a dorsal approach was used. A craniectomy was accomplished over the motor area. Then a small portion of the medial cerebral cortex was removed until, with gentle retraction, the corpus callosum could be visualized. A small opening was next made in the corpus callosum exposing the easily identified bodies of the fornices. Using a specially prepared hooked knife, the bodies of the fornices were lifted away from the

1. This work was supported by National Science Foundation Grant G-11433 for which the authors express their appreciation. 
thalamus, and then sectioned. In one additional animal, the opening was made more anteriorly, and the lesion produced by aspirating the septal area and the anterior columns of the fornix.

In two cases, electrodes were placed stereotaxically in the septal areas. Following stimulation of the septal area for another study, electrocoagulation was carried out at the tip of the electrode placements.

In each of the remaining seven animals, a craniectomy was performed over the superior part of the temporal lobe of the brain. After exposure of the superior and middle temporal gyri, an opening was made through these gyri, using the superior temporal fissure as a guide. This procedure was followed until the temporal hom of the lateral ventricle was opened and the hippocampus could be seen as the ventromedial wall of the ventricle. Electrodes were then placed visually and the hippocampus explored by electrical stimulation, with results which will be presented in another paper. Following this procedure, lesions were made in the hippocampus using either an aspirator or a special dissection instrument. This procedure was carried out in two stages, one for each side, with an interval of from 2-11 weeks. In four of these seven animals, this was the only procedure. In the other three, the procedure just described was preceded by a fornicotomy as outlined previously. Thus, in four animals there were bilateral lesions of Ammon's horn, and in three animals there were bilateral Iesions of Ammon's horn plus an additional section of the fornix bilaterally.

After survival periods of from 21 days to 3.5 months, the animals were killed by an overdose of evipal and perfused intracardially with $10 \%$ formalin. The brains were removed immediately and additionally fixed for a period of not less than two weeks in $10 \%$ formalin. They were then sectioned in $2 \mathrm{~mm}$ slices which were left in the fixative fluid for an additional week. They were then stained according to the Marchi technique as modified by Swank and Davenport ('35). Sections were cut at 30 to $50 \mu$ depending on the friability of the tissues. Every fifth section was mounted and studied microscopically. The intermediate sections were stored, and if further clarification was necessary, they were mounted and examined.

Precautions were taken to eliminate the artifacts which are inherent in the Marchi technique. However, a small proportion of normal fibers were stained, and there was the appearance of some "Marchi dust" in the sections. The only degeneration to be described will be that which could be followed serially from the lesion, and which had the characteristic black irregular shapes of Marchi degeneration. Terminal degeneration is not revealed by this method, and, accordingly, nothing but the most tentative suggestion will be made about the exact termination of the fibers to be described. Primary concern in this study is with the make-up of the fornix. Therefore, detailed descriptions of the degeneration in the projections of the fornix system which have been presented elsewhere (Votaw, '60b) will not be given here, and reference will be made to those results only for comparison.

The term hippocampal formation (or hippocampal complex), as it is used in the literature, includes the dentate gyrus, cornu Ammonis, and subiculum. At times the term has been extended to include the presubiculum and even the entorhinal area. The term hippocampus is usually used to indicate the cornu Ammonis alone although some earlier authors have used it as synonymous with hippocampal formation. In this study, the term hippocampal formation (or hippocampal complex or hippocampal area) will be used to indicate the complex of dentate gyrus, Ammon's horn and subiculum. The presubiculum and entorhinal area will be treated as separate entities. The terms hippocampus and cornu Ammonis (Ammon's horn) will be used interchangeably.

\section{RESULTS}

Lesions of septal nuclei. In one case there was complete removal of the medial and lateral septal nuclei of the left side. This lesion also interrupted the column of the homolateral fornix and slightly injured the column of the other side. The anterior commissure was involved and degeneration was observed within it. In addition, there were the lesions in the corpus callosum and in the frontal lobe. 
In the other two cases there were small circumscribed lesions bilaterally, but asymmetrically located in the medial and lateral septal nuclei. The electrode paths could be followed to the area of the lesion, but the amount of degeneration about them was insignificant. The anterior commissure and the anterior columns of the fornix were not involved in these lesions.

The degeneration resulting from these lesions tended to be largely ipsilateral, as can be determined from the first case. However, there was a significant number of degenerated fibers on the contralateral side. Degeneration could be followed in a posterior direction in the bodies of the fornices. This degeneration was somewhat diffusely dispersed throughout the body of the formix but the region of heaviest concentration was found to be on the lateral side of the fornix. Anteriorly, where the fornix forms a compact, circular bundle, the degeneration was found in the lateral half (see Votaw, '60, fig. 2b). More posteriorly, where the fornix begins to flatten out into a semi-sheet of fibers, the degeneration was diffuse but more concentrated in the lateral part of the bundle.

From this position, the degeneration continued into the fimbria along the medial side of the hippocampus. Degeneration could be seen over the entire extent of the hippocampus, reaching its most anterior portions. The concentration of degenerated tissue, however, was much more dense in the posterior regions, and gradually decreased as the pes hippocampi were reached. At all levels of the hippocampal formation, degenerated fibers could be seen to turn out from the fimbria and to enter the alveus bundle. Also, on careful observation, some degenerated fibers could be seen turning out of the alveus and entering stratum oriens of Ammon's horn.

Small granules could be seen throughout the pyramidal cell layer of Ammon's horn and the polymorphic cell layer of the dentate gyrus, as well as a few such granules in the polymorphic cell layer of Ammon's horn. Many of these granules looked like Marchi dust. It was also noticed that brains prepared according to the same technique but for another study in which the hippocampal forma- tion should not be involved at all, have a large amount of such dust. Therefore, it is not known to what position in the hippocampal formation the fibers under study go. Since there was no degeneration beyond the region of the hippocampal formation (subiculum, presubiculum, hippocampal gyrus), it is assumed that the fibers terminated somewhere in that formation.

In all three of these cases, but especially in the case in which the septal nuclei were entirely removed, degeneration can be followed in the postcommissural fornix column to the medial mammillary nucleus and other hypothalamic nuclei. No postmammillary degeneration could be found. Some degeneration was found as well in the diagonal band of Broca and a very few degenerated fibers were observed in the lateral region of the hypothalamus. The latter were thought to be in the medial forebrain bundle but the data at this time are insufficient to draw any conclusions.

Section of the fornix. (Figs. 1A, 2A, 3A.) In three cases the bodies of the fornices were completely severed bilaterally. In two of these, the section was midway between the hippocampal commissure and the septal nuclei. In the third, the lesion destroyed, in addition to the bodies of the fornices, the anterior columns bilaterally and involved the septal area to some degree (fig. 1A). To be sure, there are some fibers which cross just posterior to the septal nuclei as described by McLardy ('55a); however, it was evident from the examination of these brains that the main intrahippocampal commissure was left intact. In a fourth animal, the lesion involved only the lateral two-thirds of the body of the right fornix

In addition, these brains had a large lesion in the corpus callosum. However, although the degeneration from this lesion sometimes extended above the hippocampal commissure, at no time was there evidence of degeneration within this latter commissure. There was, in addition, a unilateral lesion in the medial wall of the frontal lobe. These animals had varying degrees of paralysis of the lower extremity opposite the side of the lesion.

Posterior to the section, degenerated fibers could be seen on the lateral side of 


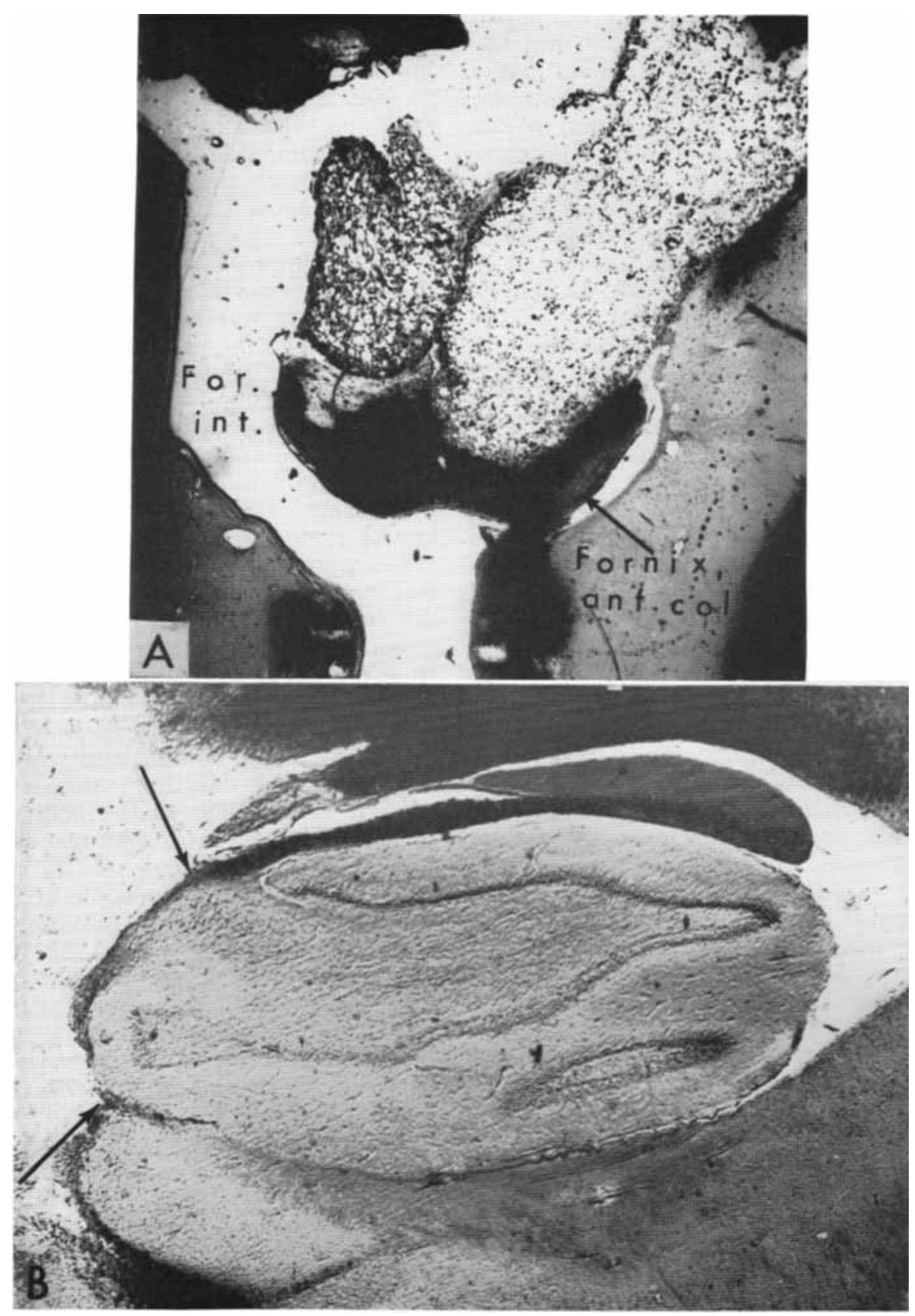

Fig. 1 Illustrations of two of the lesions described in the text. (Marchi stain; frontal sections; $X$ 12) (A) Lesion located at the posterior edge of the arch of the postcommissural fornix column. The entire vertical column of the fornix has been interrupted. For. int.: foramen interventriculare; Fornix, ant. col.: fornix, anterior column. (B) Lesion located in the hippocampus, showing the removal of the lateral layers of this structure, Arrows indicate extent of lesion. 
the body of the fornix, passing the lateral side of the region of the hippocampal commissure, and continuing into the fimbria and alveus. The place of termination of these fibers is also in doubt; the findings were the same as those described above for the septal lesions (figs. 2A, 3A).
In contrast, anterior to the lesion degeneration was distributed in the medial regions of the circular body of each fornix. Unlike the more diffusely distributed fibers in the fornices posterior to the lesion, this degeneration was confined to the medial half of the body of each fornix.

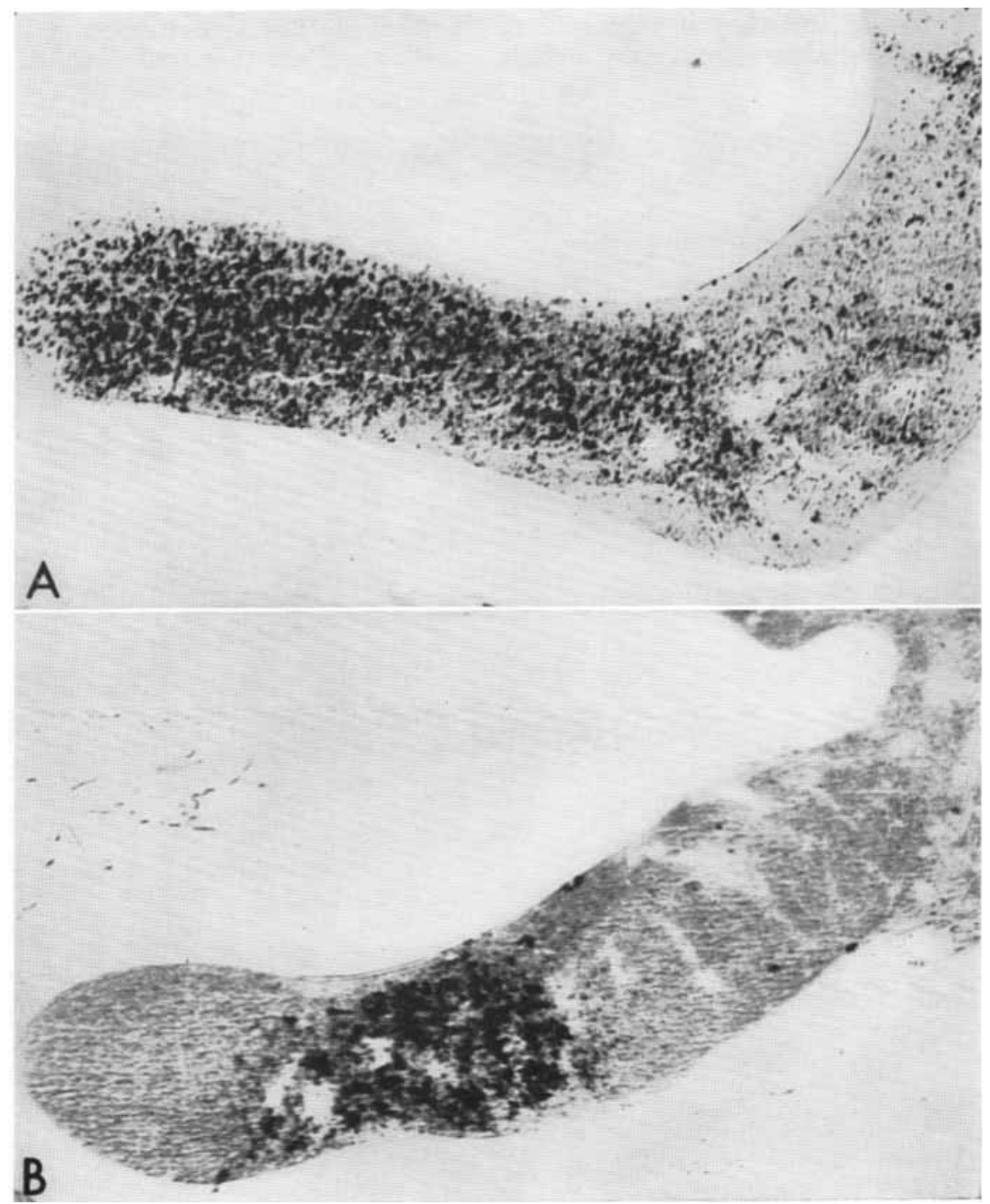

Fig. 2 Illustrations of the bodies of the fornices in the two animals whose lesions are shown in figure 1. (Marchi stain; frontal sections; $\times 27$ ) (A) Fornix, anterior to hippocampal commissure, from the animal whose lesion is shown in figure $1 \mathrm{~A}$. Note the degeneration granules are located diffusely throughout the body of the fornix with some increase in concentration in the lateral portion of the pathway. (B) Fornix in same location as $A$, but from the animal whose lesion is shown in figure 1B. Nate the localization of the degeneration in the center portion of the fiber bundle. 
Degeneration could be followed anteriorly to the region of the septal nuclei where degeneration granules were found in the lateral septal nuclei. The large columns of the fornices which course through the caudal part of the medial septal nuclei had a high concentration of degenerated tissue within them. Terminal degeneration could also be found in the diagonal band of Broca, the medial mammillary body, the hypothalamus, etc., the details of which are described elsewhere (Votaw, '60b).

Commissural fibers of the corpus callosum were degenerated and could be followed to symmetric points on each side of the brain. The pyramidal tract was heavily degenerated on the side of the lesion and its fibers could be traced through the internal capsule, the peduncles, and the pyramids. The spinal cords of these animals were not prepared according to the

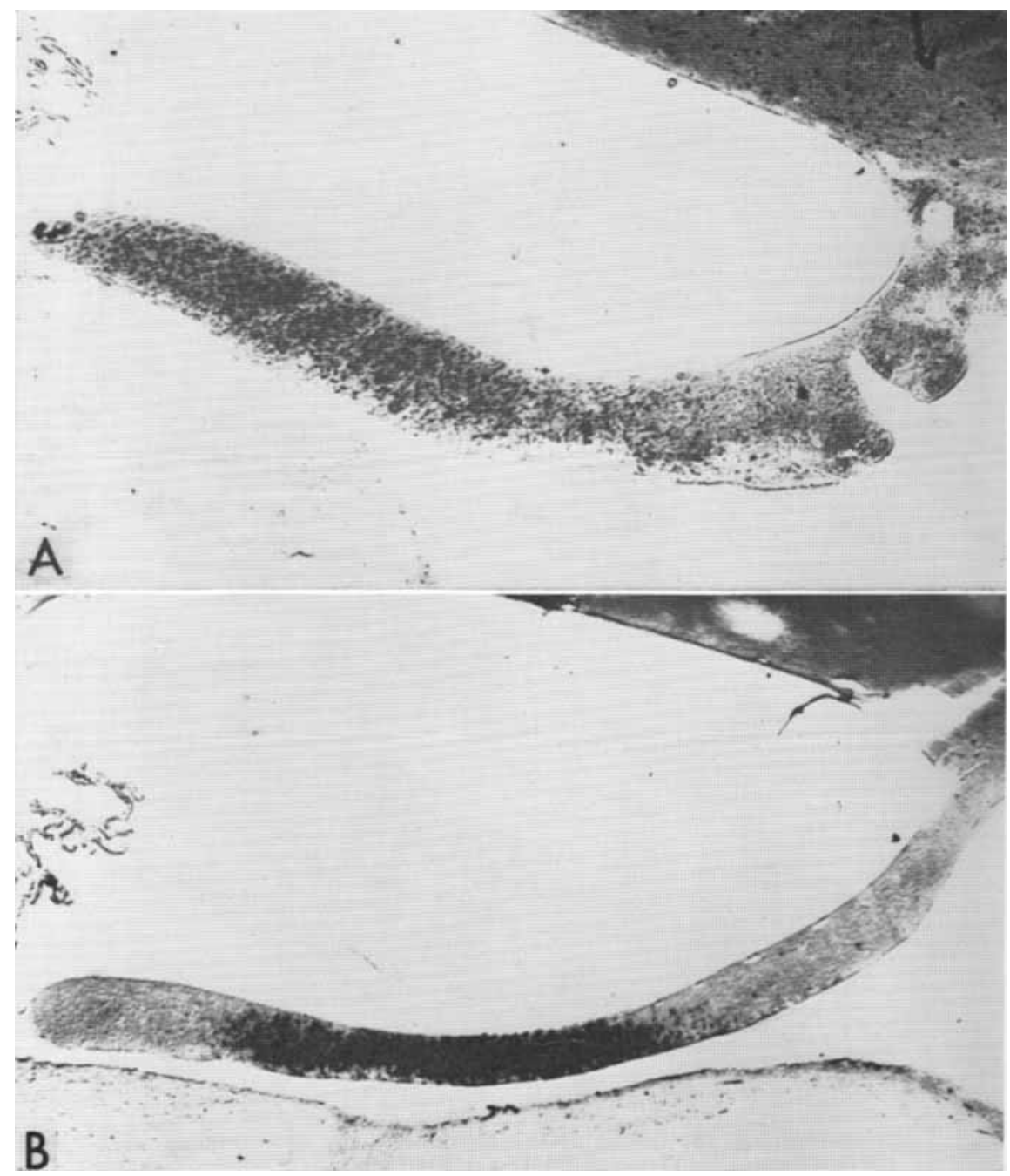

Fig. 3 Illustrations of the fornices of the same two animals at almost the junction of the body of the fornix with the fimbria. (Marchi stain; frontal sections; $\times 23$.) (A) Fornix from animal with lesion shown in figure 1A. (B) Fornix from animal with lesion shown in figure 1B. Again note the distribution of the degeneration granules. 
Marchi technique, and thus degeneration was not examined further than midmedulla regions.

Lesions of Ammon's horn. (figs. 1B, 2B, 3B.) These lesions involved the outer layers of Ammon's horn (the alveus, the pyramidal cells, and the polymorphic cells) (fig. 1B) and extended for about three-fourths of the length of the hippocampus. The lesions involved the areas CA-1, CA-2, CA-3, but only a small part of the continguous $\mathrm{CA}-4$ and prosubiculum. The fimbria and the entire dentate gyrus escaped injury.

From the borders of the lesions degenerated fibers could be traced into the alveus (fig. 1B). Some of these fibers went across the alveus to the fimbria where they turned caudally and then superiorly along the length of the hippocampus; others ran at a more oblique angle along the hippocampus in the alveus bundle and entered the fornix at the termination of Ammon's horn. On reaching the arch of the fornix, these degenerated fibers could be seen on the medial side of the formix body (fig. 3B). In the hippocampal commissure there was a high concentration of fibers which contained degeneration granules. The fibers that did not cross proceeded rostrally through the body of the fornix on its medial side (fig. 2B). They did not appear to be diffusely arranged, and there was practically no degeneration of the lateral side of the fornix body.

These fibers continued forward to the septum, where there was a large amount of degeneration in the lateral septal nucleus, and some in the medial septal nucleus. Some fibers entered the precommissural fornix and went to the diagonal band of Broca. The presence of degenerated fibers in the postcommissural fornix, the stria medullaris thalami, or any of the thalamic or hypothalamic nuclei could not be established. No degenerated fibers could be traced into the midbrain from the fornix structures. On the contrary, the postcommissural fornix appeared entirely normal in these preparations.

Temporotegmental, temporopontine, and temporopulvinar fibers could be followed from the region of the temporal lobe lesions to their respective terminations.
These pathways were distinct from those described above.

\section{Lesions of Ammon's horn plus section of the fornix}

In three animals the fornices were sectioned bilaterally. Later examination demonstrated that both fornices had been completely sectioned at a point about onethird the distance from the septal nuclei to the hippocampal commissure. The commissure was not involved in any of these lesions. After recovery from the fornix section operation, one hippocampus was exposed and a lesion placed in it. A similar destruction was produced in the hippocampus of the other side at a still later date. The destruction in the temporal lobe and Ammon's horn was comparable to that described in the previous group of experiments. Again, the dentate gyrus and the fimbria escaped direct injury.

Degeneration anterior to the section of the fornices was similar in all respects to that described for the second group of lesions. Similarly, degeneration from the temporal lobe lesions in diencephalic and brain stem regions was the same as that described in the previous group of lesions. The hippocampal commissure was markedly, though not completely, degenerated.

In these cases the one significant finding was in the body of the fornix posterior to the section in this structure. This region lies between two lesions which have been described as producing characteristically located degeneration in the bodies of the fornices. Examination of the fornix anterior to the hippocampal commissure showed that the entire fornix contained degenerated fibers, with a heavy concentration medially, and somewhat more diffusely distributed degeneration in the lateral portion of this fiber bundle.

In summary, (fig. 4) it has been noted that lesions of the septal nuclei and the anterior part of the bodies of the fornix give rise to degenerated fibers which appear to run caudally in the lateral portions of the fornix. This degeneration is somewhat diffusely distributed, with heavier concentration in the lateral part of the body of the fornix. These fibers continue past the hippocampal commissure, enter the fimbria, then the alveus, and appear 


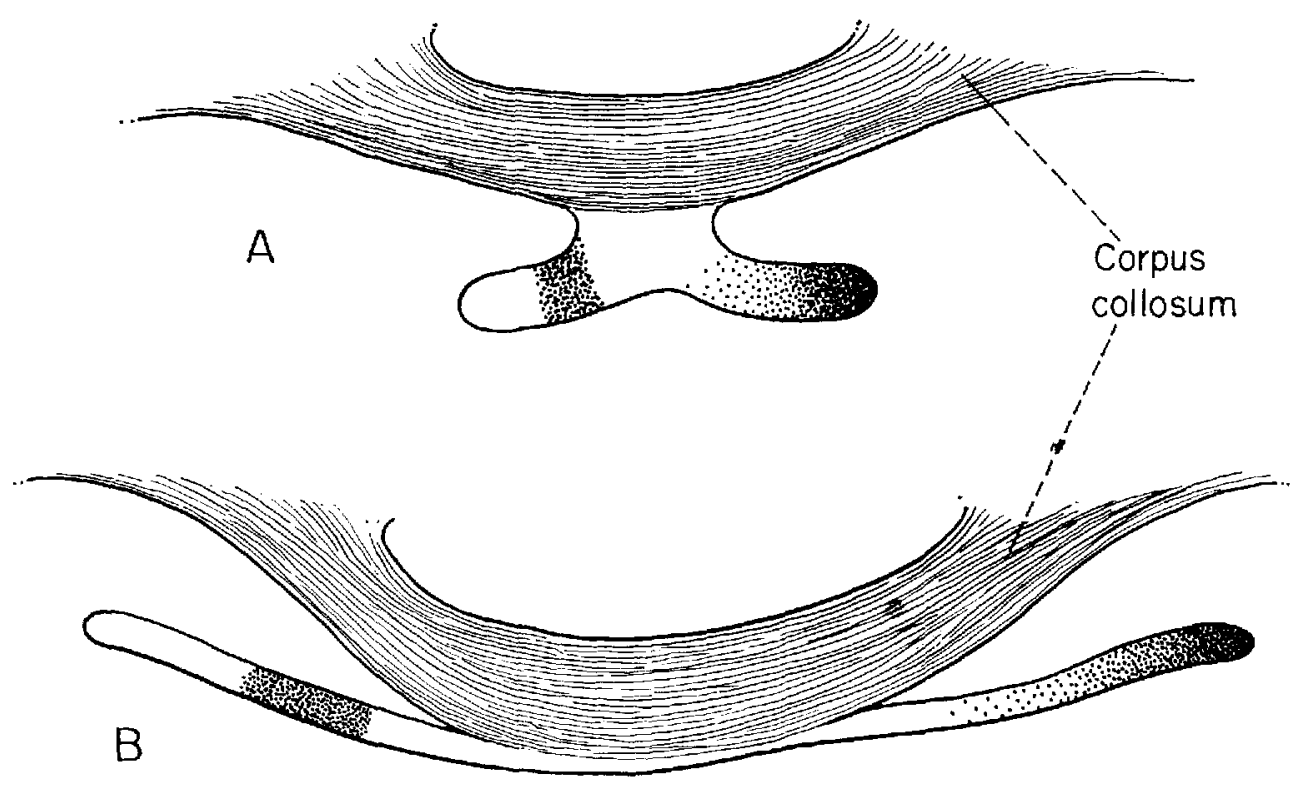

Fig. 4 Diagrammatic illustrations of frontal sections of the fornix at levels (A) of the anterior thalamic nucleus and (B) of the habenula. The right side of the drawings shows the location of degenerating fibers following septal lesions; the left side, the location after hippocampal lesions.

to turn into Ammon's horn and the dentate gyrus at all levels of the hippocampal formation. The degeneration is bilateral with a much heavier concentration of degeneration granules on the side of the lesion.

Lesions in Ammon's horn which do not involve the fimbria or the dentate gyrus give rise to degenerated fibers which run caudally along the hippocampus and then enter the fornix on its medial side, with many of the fibers crossing over in the hippocampal commissure. Those which do not cross run forward in the medial portion of the body of the fornix to terminate in anterior regions of the rhinencephalon, namely, the septal nuclei and the diagonal band of Broca.

It is further noted that lesions in Ammon's horn, which do not destroy a part of the dentate gyrus, produce a different terminal distribution of degeneration than that which results from section of the fornix. When the fornix is sectioned, degeneration occurs in the stria medullaris thalami, postcommissural fornix, hypothalamic nuclei, etc., but degeneration secondary to lesions in Ammon's horn alone appears to be restricted to the anterior parts of the limbic lobe.

\section{DISCUSSION}

There has been an increasing awareness of the fact that there are hippocampal afferent fibers in the fornix. Prior to the time of Ramón y Cajal, and, indeed, in many more recent descriptions of the brain, the fornix is described as a band of fibers running from the diencephalon to the hippocampus. Ramón y Cajal ('11) described the course of the fornix fibers as running in the opposite direction toward the mammillary body. It has been assumed by many, since that time, that the fornix is the only, and entirely, efferent outlet of the hippocampal formation. Brodal ('47) repeats this suggestion in his excellent review of the hippocampus and the sense of smell, but leaves room for the possibility that there are septohippocampal fibers, and that there might be conduction in a direction opposite from that which had been generally assumed.

Crosby ('17) on the basis of her description of the normal anatomy of the reptile, suggested that the medial septal 
nucleus might be a way station for ascending impulses to the hippocampus. Loo ('31), Young ('36), Fox ('43), and Lauer ('45) all made similar suggestions from the study of normal material of the opossum, rabbit, cat, and monkey, respectively. However, these were all descriptions of normal material prepared for microscopic study, and did not incorporate experimental proof of the direction of conduction.

Mettler ('43) noted in the monkey and Rose and Woolsey ('43) in the rabbit that massive ablations of the cerebral hemispheres did not result in any retrograde cellular damage to the septal nuclei unless the hippocampus was included. Other retrograde cell degeneration studies by Sprague and Meyer ('50) in the rabbit, Daitz and Powell ('54) in the rat, and McLardy ('55a) in the monkey showed that lesions of the fornix resulted in rather marked retrograde cell degeneration of the medial septal nucleus, implying the presence of caudally running fibers in the fornix.

Gerebtzoff ('39, '41) suggested that the dorsal fornix of the guinea pig contained fibers having their cells of origin in the diagonal band of Broca and which terminated in Ammon's horn. Cragg and Hamlyn $(' 57,59)$ found fibers in the dorsal fornix, arising from the septal nuclei of the rabbit, and terminating in the presubiculum but not in Ammon's horn. Morin ('50), using the guinea pig, noted that lesions in the precommissural system ventral to the genu produced Marchi degeneration in the fornix which went posteriorly as far as the hippocampal commissure and the fimbria. Guillery ('57) and Cragg ('61) placed lesions in the hypothalamus of the rat, rabbit, and cat and then studied the resulting degeneration with the Nauta-Gygax technique. They observed fibers ascending in the medial forebrain bundle, some of which bypassed the septal area, entered the fornix, and went to the hippocampus. Finally, in a study of the connections of the cingulum in the albino rat, White ("59) noted that degeneration appeared in the hippocampus only when damage to the septal area, fornix, or dorsal psalterium was evident in his specimens. Cus and Stern ('61) were equally convinced of a relationship be- tween the septal area and hippocampus of the rat.

The evidence presented here strongly suggests a hippocampal afferent fiber system in the fornix of the monkey. From previous accounts and from the data presented here, the origin of these fibers may be the septal area (specifically, the medial septal nuclei) or the hypothalamic nuclei which give off fibers which pass by way of the medial forebrain bundle to enter the fornix. The lesions of this study were not in the proper location to determine the origin of the hippocampal afferent fibers. McLardy ('55a, '55b) suggested that there are fibers in the fornix, some of which cross the midline just posterior to the septal area, and interrelate the septal nuclei and the isocortex near the anterior part of the temporal lobe. He also described these fibers as present in the medial part of the fornix. The fibers extending caudally in the fornix as described here are more characteristically located on its lateral side. However, unlike the rostrally running fibers in the fornix, which were rather definitely located on the medial side of this bundle, the posteriorly coursing fibers were more diffuse, being more concentrated on the lateral side, but with some degeneration on the medial side. These medial fibers could be the same as those described by McLardy. No degeneration was observed in the temporal cortex, but this failure may be due to the technique used. McLardy's data depended on retrograde cell degeneration. Certainly, the observations presented here do not contradict the evidence of a septotemporal pathway as described by McLardy, but a much heavier lateral degeneration in the fornix, resulting from septal and fornix lesions, passes caudally in the fornix. The difference between the results of this study and that of McLardy's may be due to one of technique. A temporoseptal interconnection has been confirmed by Stoll, Ajmone-Marsan, and Jasper ('51) using electrophysiological techniques.

Electroneurophysiological methods are perhaps somewhat more convincing than are the morphological studies in demonstrating a hippocampal afferent fiber system in the fornix. In a very careful and thorough analysis of the electrical activity 
of the hippocampus as a result of stimulation of the fornix, reticular system, and peripheral nerves by Green and co-workers (Green and Morin, '53; Green and Arduini, '54; Green and Adey, '56; Von Euler, Green and Ricci, '58), it was shown that there is a response in the hippocampus to stimulation of the fornix, reticular system, and peripheral nerves. They further demonstrate that this activity is orthodromically produced. Stimulation of the reticular system produced a peculiar type of arousal activity in the hippocampus. This arousal activity was eliminated by septal lesions which also interrupted the fornix system.

From a similar study, Fujika and Nakamura ('61) have concluded that there are both excitatory and inhibitory afferent systems in the fornix going to the hippocampus. They do not demonstrate to their own satisfaction that the excitatory influence is a strictly orthodromic one. The possibility exists that antidromic stimulation of the pyramidal cell axons orthodromically stimulates the collaterals of this axon (Schaeffer collaterals) which, in turn, can orthodromically cause a postsynaptic response. However, Green and his co-workers (quoted above) and recently Feldman ('62) have shown that stimulation of more distant structures (hypothalamus, reticular system, peripheral nerves) results in excitatory influences in the hippocampus which may reach it by way of the formix.

Andersen and co-workers (Andersen, Bruland and Kaada, '61a, '61 b) and Dunlop ('58) have carried out electrophysiologic studies on the reactions of the hippocampal pyramids secondary to stimulation of the septum and/or fornix. They find excitatory responses that must be carried along hippocampal afferent fibers.

Adey, Sunderland and Dunlop ('57) stimulated the fornix and found that, in the marsupial, they could obtain responses in the hippocampus, followed by responses in the entorhinal area. These responses were easily elicited. In contrast, it was much more difficult to stimulate the entorhinal area and record a response in the fornix (the more classical direction of conduction). Frequently, this latter response could not be obtained at all. Cragg and Hamlyn ('57) found that stimulation of the septum in rabbits caused a response in the presubiculum which was then followed by a response in the hippocampus. They did not find any direct septohippocampal responses. In this particular species, they found that lesions of the septum produce degeneration (NautaGygax technique) in the presubiculum, but they did not report any in the hippocampus. Similar morphological results were found later by these authors in the same species ('59). Certainly the preponderance of the data in the literature suggests that there is conduction from the fornix to Ammon's horn and to the entorhinal cortex. Blackstad ('56, '58) has reported commissural fibers in the fornix that project to the presubiculum and other parts of the hippocampal complex, and perhaps some of these fibers were involved in Cragg and Hamlyn's work, although from the description of the technique in the latter investigation, it would not seem so.

Rose and Woolsey ('49) have distinguished the Ammon's horn and entorhinal area as the only cortical fields which do not receive thalamic fibers, a fact that would set apart this portion of the telencephalon. That Ammon's horn is not solely related to olfaction has been well shown (see Brodal, '47, Votaw, '59, for further references). It has been suggested that perhaps the hippocampus has some influence on other cortical fields. The ascending influences (hippocampocortical) may well rely on the information which the hippocampus receives through the afferent fibers in the fornix. As the thalamus does not appear to project to the hippocampus, and, from the data in the literature quoted here, the hypothalamus and reticular areas (as well as others) do, the hippocampal system may well be set apart physiologically as a separate system with a function that is poorly understood.

Finally, the difference observed in this study between lesions of the fornix and lesions of the layers of Ammon's horn where the dentate gyrus and fimbria were not involved should be noted. Section of the fornix produced Marchi degeneration in all of the classical terminations of the fornix system ( see Nauta, '56; Brodal, '47; 
Valenstein and Nauta, '59 and Ramón y Cajal, '11). Lesions of Ammon's horn which did not include dentate gyrus and fimbria resulted in Marchi degeneration products in the anterior parts of the fornix system (septal area, diagonal band of Broca, commissural fibers) but little evidence could be found for degeneration in the postcommissural parts of the fornix projection system. The data are not complete enough to reach any definite conclusions, but it is suggested that perhaps there are marked differences in the pattern of termination of the projection fibers of the dentate gyrus as compared to those of Ammon's horn, and that these may be important from a physiological standpoint.

In as much as the dentate gyrus was not involved in the lesions discussed here, it might be assumed that fibers arising in this gyrus would be found in lateral parts of the bodies of the fornix. However, complete section of the fornix bilaterally which would have cut such fibers (as the two cases reported in the second group of animals in the results ) still did not result in degeneration of the lateral portion of the fornix. We must conclude that most of the lateral portion of the fornix is reserved for hippocampal afferent fibers.

\section{CONCLUSIONS}

1. A study of the components of the fornix of the monkey has been accomplished by placing selected lesions in the septal area, fornix, and Ammon's horn and investigating the resulting degeneration as demonstrated by the Marchi technique.

2. It was found that there is a hippocampal afferent fiber system in the fornix, located on the lateral side of the body of the fornix, which arises from anterior areas (septum suggested) and terminates somewhere in the hippocampal formation.

3. The well-known hippocampal efferent system arising from Ammon's horn is located on the medial side of the body of the fornix and terminates in the anterior projection field of the formix system.

4 . The relation of these findings to previous ones in the literature is discussed.

\section{LITERATURE CITED}

Adey, W. R., S. Sunderland and C. W. Dunlop 1957 The entorhinal area; electrophysiological studies of its interrelations with rhinen- cephalic structures and the brain stem. EEG Clîn. Neurophysiol., 9: 309-324.

Andersen, P., H. Bruland and B. R. Kaada 1961a Activation of the dentate area by septal stimulation. Acta Physiol. Scand., 51: 17-28.

1961b Activation of the field CA-1 of the hippocampus by septal stimulation. Ibid., 51: $29-40$.

Blackstad, T. W. 1956 Commissural connections of the hippocampal region in the rat, with special reference to their mode of termination. J. Comp. Neur., 105: 417-538.

- 1958 On the termination of some afferents to the hippocampus and fascia dentata. Acta Anat., 35: 202-214.

Brodal, A. 1947 The hippocampus and the sense of smell. Brain, 70: 179-224.

Cragg, B. G. 1961 Olfactory and other afferent connections of the hippocampus in the rabbit, rat, and cat. Exp. Neurol, 3: 588-600.

Cragg, B. G., and L. H. Hamlyn 1957 Some commissural and septal connexions of the hippocampus in the rabbit. A combined histological and electrical study. J. Physiol., 135: $460-485$.

1959 Histologic connections and electrical and autonomic responses evoked by stimulation of the dorsal fornix in the rabbit. Exp. Neurol., 1: 187-213.

Crosby, E. C. 1917 The forebrain of Alligator mississippiensis. J. Comp. Neur., 27: 325-402.

Cus, M., and P. Stern 1961 Beitrag zur funktionellen Beziehung des Hippocampus und Septum pellucidum bei der Ratte. Z. ges. exp. Med., 134: 443-445.

Daitz, H. M., and T. P. S. Powell 1954 Studies of the connexions of the fornix system. J. Neurol. Neurosurg. Psychiat., 17: 75-82.

Dunlop, C. W. 1958 Viscero-sensory and somato-sensory representation in the rhinencephalon. EEG Clin. Neurophysiol., 10: 297304.

Feldman, S. 1962 Neurophysiological mechanisms modifying afferent hypothalamo-hippocampal conduction. Exp. Neurol., 5: 269-291.

Fox, C. A. 1943 The stria terminalis, longitudinal association bundle and precommissural fornix fibers in the cat. J. Comp. Neur., 79: 277-291.

Fujika, Y., and Y. Nakamura 1961 Effect of fornical stimulation upon the CA-1 and CA-2 apical dendrite of rabbit's hippocampus. Jap. J. Physiol., 11: 357-368.

Gerebtzoff, M. A. 1939 Sur quelques voies d'association de l'écorce cérébral. J. Belge Neurol., 39: 205-221.

1941 Note anatomo-expérimentale sur le fornix, la corne d'Ammon et leur relations avec diverse structure encéphaliques notamment l'épiphyse. Ibid., 41: 199-206.

Green, J. D., and W. R. Adey 1956 Electrophysiological studies of hippocampal connections and excitability. EEG Clin. Neurophysiol., 8: 245-262.

Green, J. D., and A. Arduini 1954 Hippocampal electrical activity in arousal. J. Neurophysiol., 17: 533--554. 
Green, J. D., and F. Morin 1953 Hypothalamic electrical activity and hypothalamo-cortical relationships. Amer. J. Physiol, 172; 175-186.

Guillery, R. W. 1957 Degeneration in the hypothalamic connexions of the albino rat. $\mathrm{J}$. Anat., 91: 91-116.

Lauer, E. W. 1945 The nuclear pattern and fiber connections of certain basal telencephalic centers in the macaque. J. Comp. Neur., 82: 215-254.

Loo, Y. T. 1931 The forebrain of the opossum Didelphis virginiana. Part II. Histology. Ibid., 52: 1-148.

McLardy, T. 1955a Observations on the fornix of the monkey. I. Cell studies. Ibid., 103: 305-325.

1955b Observations on the fornix of the monkey. II. Fiber studies. Ibid., 103: 327343.

Mettler, F, A. 1943 Extensive unilateral cerebral removals in the primate: physiologic effects and resultant degeneration. Ibid, 79: $185-245$.

Morin, F. 1950 An experimental study of hypothalamic connections in the guinea pig. Ibid., 92: 193-213.

Nauta, W. J. H. 1956 An experimental study of the fornix in the rat. Ibid., 104: 247-272.

Ramón y Cajal, S. 1911 Histologie du système nerveux de l'homme et des vertébrés. A. Maloine, Paris.

Rose, J. E., and C. N. Woolsey 1943 A study of thalamo-cortical relations in the rabbit. Bull. Johns Hopkins Hosp., 73: 65-128.

- 1949 Organization of the mammalian thalamus and its relationships to the cerebral cortex. EEG Clin. Neurophysiol., 1: $391-404$.
Sprague, J. M., and M. M. Meyer 1950 An experimental study of the fornix in the rabbit. J. Anat., 84: 354-368.

Stoll, J., C. Ajmone-Marsan and H. H. Jasper 1951 Electrophysiological studies of subcortical connections of anterior temporal region in cat. J. Neurophysiol., 14: 305-316.

Swank, R. L., and H. A. Davenport 1935 Chlorate-osmic-formalin method for staining degenerating myelin. Stain Technol, 10: 87-90.

Valenstein, E. S., and W. J. H. Nauta 1959 A comparison of the distribution of the fornix system in the rat, guinea pig, cat, and monkey. J. Comp. Neur., 113: 337-363.

Von Euler, C., J. D. Green and G. Ricci 1958 The role of hippocampal dendrites in evoked responses and after-discharge. Acta Physiol. Scand., 42: 87-111.

Votaw, C. L. 1959 Certain functional and anatomical relations of the cornu Ammonis of the macaque monkey. I. Functional relations. J. Comp. Neur., 112: 353-382.

_ 1960a Study of septal stimulation and ablation in the macaque monkey. Neurol., 10: 202-209.

- $1960 \mathrm{~b}$ Certain functional and anatomical relations of the cornu Ammonis of the macaque monkey. II. Anatomical relations. J. Comp. Neur., 114; 283-293.

White, L. E. 1959 Ipsilateral afferents to the hippocampal formation in the albino rat. Ibid., 113: 1-42.

Young, M. W. 1936 The nuclear pattern and fiber connections of the noncortical centers of the telencephalon of the rabbit (Lepus cuniculus). Ibid, 65: 295-401. 